\title{
MULTIVARIATE REGRESSION APPLIED TO THE PERFORMANCE OTIMIZATION OF A COUNTERCURRENT ULTRACENTRIFUGE - A PRELIMINARY STUDY.
}

\begin{abstract}
E. Migliavacca ${ }^{a}$, ABSTRACT
and D. A. Andrade ${ }^{a}$

a, Instituto de Pesquisas Energéticas e Nucleares

Av. Prof. Lineu Prestes, 2242 Cidade Universitária

CEP 05508-970 São Paulo SP

Tel.: 3817-7599

IPEN/CNEN

In this work, the least-squares methodology with covariance matrix is applied to determine a data curve fitting in order to obtain a performance function for the separative power $U$ of an ultracentrifuge as a function of variables that are experimentally controlled. The experimental data refer to 173 experiments on the ultracentrifugation process for uranium isotope separation. The experimental uncertainties related to the independent variables measurements are considered in the calculation of the experimental separative power values, determining an experimental data input covariance matrix. The process control variables, which significantly influence the $U$ values, are chosen in order to give information on the ultracentrifuge behaviour when submitted to several levels of feed flow $F$ and cut . After the model goodness-of-fit validation, a residual analysis is carried out to verify the assumed basis concerning its randomness and independence and mainly the existence of residual heteroscedasticity with any regression model variable. The response curves are made relating the separative power with the control variables $F$ and , to compare the fitted model with the experimental data and finally to calculate their optimized values.
\end{abstract}

\section{NOMENCLATURE}

$F \quad$ feed flow rate, a.u.

$P$ product flow rate, $a . u$.

$W \quad$ waste flow rate, $a . u$.

$z \quad$ isotope desired composition in feed F, a.u.

$y \quad$ isotope desired composition in product $\mathrm{P}$, a.u.

$x \quad$ isotope desired composition in waste $\mathrm{W}, a . u$.

$R_{p} \quad$ abundance ratio of the product.

$R_{w} \quad$ abundance ratio of the waste.

$R_{f} \quad$ abundance ratio of the feed.

$n \quad$ size of the sample

$L$ number of attributes of the total uncertainty.

$e \quad$ partial uncertainty magnitude.

$Y \quad$ generic dependent variable.

$x_{i}, x_{j} \quad$ generic controlled variables.

$V \quad$ covariance matrix.

$A \quad$ project matrix

$P p \quad$ pressure, a.u.

\section{Greek symbols}

$\alpha \quad$ independent variable standard deviation

$x^{2} \quad$ chi-square

$x^{2} \mathrm{r}_{e d} \quad$ normalized chi-square

$\delta U$ ultracentrifuge separative power.

$p \quad$ microcorrelation between uncertainties for $\mathrm{x}_{\mathrm{i}}$ and $\mathrm{x}_{\mathrm{j}}$.

$\theta \quad$ cut

$\beta_{i} \quad$ coefficients estimates

$\beta \quad$ coefficients estimates vector.

\section{Subscripts}

$i l, j l \quad$ attribute uncertainty values

ijl attribute relating uncertainty values

exp. experimental value

calc. calculated value

$p \quad$ product line

$w \quad$ waste line
Keywords: ultracentrifuge, uranium hexafluoride, isotopic separation, covariance matrix, least-squares method.

\section{$f \quad$ feed line}

\section{Superscripts}

$t \quad$ matrix transposition

\section{INTRODUCTION}

A gas ultracentrifuge, as schematized in Fig. 1 is composed of a long, thin vertical cylinder (rotor), rotating around its axis at a high velocity inside a case under vacuum.. The process gas, assumed to be a binary isotopic mixture with ${ }^{235} \mathrm{UF}_{6}$ and ${ }^{238} \mathrm{UF}_{6}$, inside the cylinder is subjected to a centrifuge force that establishes a pressure gradient in the radial direction, increasing from the center to the rotor wall (Jordan, 1980). That pressure distribution is slightly dissimilar for the different isotopes because it is proportional to mass. This results in a partial separation of the feed $F$, into two fractions: an enriched one (product) and another depleted (waste) in the desired isotope $\left({ }^{235} \mathrm{UF}_{6}\right)$. The ultracentrifuge performance and production capacity evaluation is usually done by means of the required work to isotope separation, which is proportional to the amount of processed material and to the obtained separation degree. Denoting by $F, P$ e $W$, the streams of feed, product and waste and by $z, y$ e $x$, the respective isotope desired compositions, the dependent variable that best defines the separative efficiency of any isotope separation unit, is the separative power or capacity $\delta U$, given by the following expression:

$$
\delta U=P \frac{R_{p}-1}{R_{p}+1} \ln R_{p}+W \frac{R_{w}-1}{R_{w}+1} \ln R_{w}-F \frac{R_{f}-1}{R_{f}+1} \ln R f
$$

where $F, P$ and $W$ are the operational variables and the response variables are the abundance rations of product $R_{p}=y /(1-y)$ and waste $R_{w}=x /(1-x)$. 


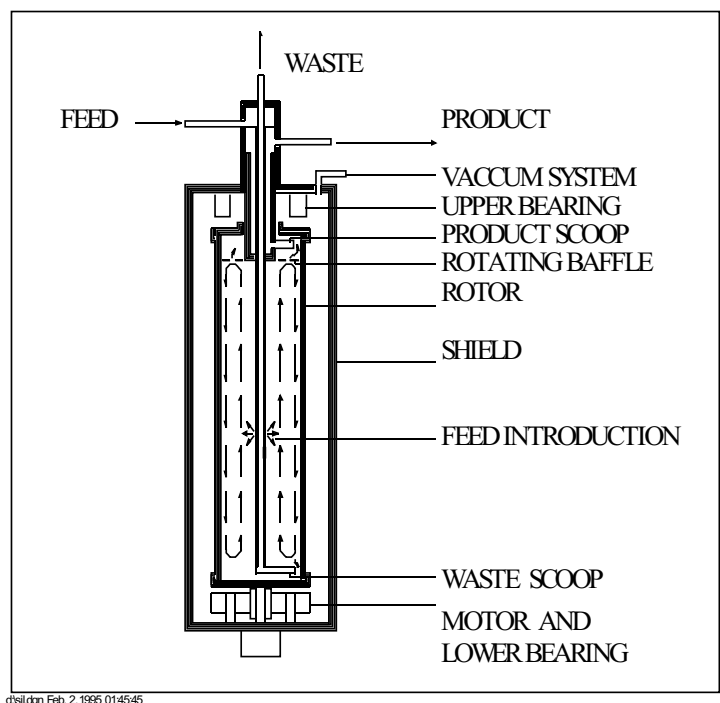

Figure 1. Countercurrent ultracentrifuge design

\section{EXPERIMENTS}

An isotopic separation test consists in the operation of an ultracentrifuge in a bench plant shown in Fig. 2. The ultracentrifuge receives an injection of a binary isotopic mixture with ${ }^{235} \mathrm{UF}_{6}$ and ${ }^{238} \mathrm{UF}_{6}$ as feed flow $F$ and permits the extractions of the product flow $P$ and waste flow $W$. Samples are collected for verification of the separation obtained by the measures of the abundance ratio of the enriched and depleted streams, $R_{p}$ and $R_{w}$, respectively, allowing to calculate the separative power $\delta U$, given by Eq. (1). Defining the cut $\theta$ as the relation between the product and feed flow and fixing the product pressure line $p_{p}$, several groups of data are generated with the variation of the cut $\theta$ and the feed flow $F$. Each of them is denominated a separation experiment, resulting in an ultracentrifuge performance function like $\delta U(F, \theta)$.

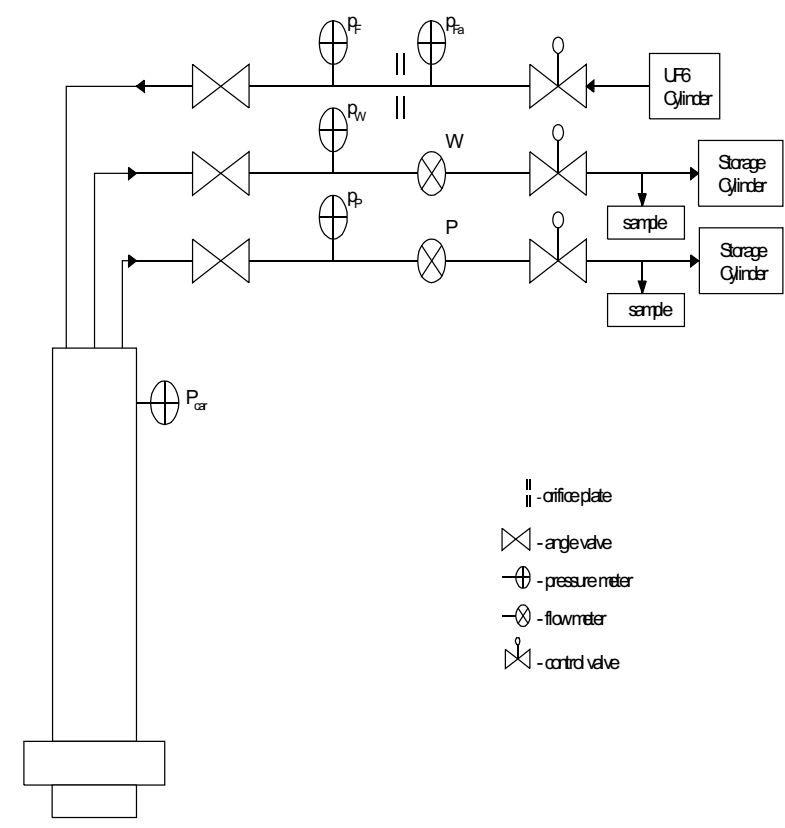

Migliavacca and Andrade et al. Multivariate regression ... STATISTICAL THEORY

The measurements of $R_{P}, R_{W}, P$ and $W$, involved in the separative power determination $\delta U$, provide correlated uncertainties and define a covariance between them. These statistical uncertainties are propagated in Eq. (1) in order to obtain the $\delta U$ final uncertainty with the expression (Cowan, 1998):

$$
\left(\sigma_{\delta U}\right)^{2} \approx \sum_{i=1}^{n}\left(\frac{\partial \delta_{U}}{\partial x_{i}}\right)^{2} \sigma_{i}^{2}
$$

where $x_{i}$ are the independent variables $R_{P}, R_{W}, P$ and $W, \sigma_{i}$ express their respective variances. The $R_{P}$ and $R_{W}$ variances are directly given by mass spectrometry analysis while the $P$ and $W$ variances are calculated from mass flowmeters calibration curves. Each $\delta U$ experimental data covariance matrix element is calculated by the expression:

$$
\left(V_{\delta U}\right)_{i j}=\sum_{l=1}^{L} \rho_{i j l} e_{i l} e_{j l} \quad(i, j=1, n)
$$

where $e_{i l}, e_{j l}$ are the partial uncertainty magnitude of any independent variable $R_{P}, R_{W}, P$ and $W ; \rho_{i j l}$ represents the microcorrelations between these variable measurements due to each attribute $l$. The process analysis permits to determine these microcorrelations values with safety. The $\delta U$ experimental data fitting through a performance function of the kind $\delta U(F, \theta)$ is obtained due to $\delta U$ and $(F, \theta)$ relation, that may be written as a second order polynomial given by:

$$
\begin{aligned}
& Y=\beta_{0}+\sum \beta_{i} x_{i}+\sum \beta_{i i} x_{i}^{2}+\sum \beta_{i j} x_{i} x_{j} \\
& +\sum \beta_{i i j} x_{i}^{2} x_{j}+\sum \beta_{i i j j} x_{i}^{2} x_{j}^{2} \quad i \neq j
\end{aligned}
$$

where $Y$ is the response $(\delta U), \quad \beta_{i}$ are the equation coefficients and $x_{i}, x_{j}$ are the controlled variables $(F, \theta)$. This equation is used to evaluate the linear, quadratic and interaction effects between these variables providing the project matrix A, that contains all the fitted model explained variables. The Eq. (4) is a linear function in the $\beta$ parameters and although we can perform the least-squares method to any function, in this case the chi-square and estimators resulting values have desired properties: the estimators and their variances can be analytically obtained, they will be unbiased with minimum variance no matter the number of experiments and the experimental data distribution function. According to the least-square method with covariance matrix, the best possible solution is the one which minimizes the chi-square $\chi^{2}$. The $\chi^{2}$ value for this particular problem is given by (Smith, 1981 and 1993):

$$
\chi^{2}=\left(\delta U_{\exp }-\delta U_{c a l c}\right)^{t} V_{\delta U}{ }^{-1}\left(\delta U_{\exp }-\delta U_{c a l c}\right)
$$




\section{Tecnologia/Technology}

where $\delta U_{\text {calc }} \approx A \beta$, and $\beta$ is the coefficients estimates vector of the fitted equation. Under the following conditions: (i) the $\delta U$ experimental data is distributed according to a normal with a known covariance matrix, which permits to use the chi-square statistic, (ii) the fitted function, Eq.(4) is linear in the coefficients $\beta i$, allowing to obtain an analytical solution for Eq.(5) and (iii) the functional form of the fitted function, Eq.(4), is corrected, , i. e., it is possible to obtain the minimum deviation between the experimental and predicted values, so the quadratic form $\chi^{2}$ should be distributed in conformity with the chisquare tables, allowing to evaluate the model goodness-offit (Cowan, 1998).

The desired least-square solution is given by:

$$
\beta=V_{\beta} A^{t} V_{\delta U}^{-1} \delta U_{\exp }
$$

where the covariance matrix for the solution $\beta$ is given by:

$$
V_{\beta}=\left(A^{t} V_{\delta U}^{-1} A\right)^{-1}
$$

That gives the coefficients estimates variances and covariances of the experimental data fitted curves. In this case, a FORTRAN program (Migliavacca, 2004) is used.

\section{RESULTS AND DISCUSSION}

The experimental data performed with only one ultracentrifuge covered the whole domain of interest, consisting of eight values of feed flow $F$, seven values of cut $\theta$ and fixing the product pressure line $p_{p}$, resulting in a group of 173 experiments. Due to secret character inherent to the process development, the sensitive data were codified, with all variables related to arbitrary units. The isotopic abundance ratios $R_{F}, R_{P}$ e $R_{W}$, and the flow values $F, P$ e $W$, with their respective uncertainties; the separative power $\delta U$ and cut $\theta$ experimental values are presented in Tab. (1). In Tab. (2), are presented the coefficients estimates of the fitted equation, the determination coefficient, the chi-square and normalized chi-square and in Tab. (3) are presented their variances and covariances in the upper triangle and their correlations in the lower triangle.

Table 1. Codified variables and their uncertainties values

\begin{tabular}{|c|c|c|c|c|c|}
\hline Exp & $\begin{array}{l}\boldsymbol{R}_{F} \\
\left(\boldsymbol{x} \mathbf{1 0} \mathbf{0}^{-04)}\right.\end{array}$ & $\begin{array}{l}\boldsymbol{R}_{p} \\
\left(\boldsymbol{x} \mathbf{1 0}^{-03}\right)\end{array}$ & $\begin{array}{l}\boldsymbol{R}_{w} \\
\left(\boldsymbol{x} \mathbf{1 0}^{-04}\right)\end{array}$ & $\begin{array}{l}\sigma_{R f} \\
\left(\boldsymbol{x}^{-06}\right)\end{array}$ & $\begin{array}{l}\sigma_{R p} \\
\left(\boldsymbol{x} \mathbf{1 0} \mathbf{0}^{-07}\right)\end{array}$ \\
\hline 1 & 9.8086 & 1.1982 & 8.9588 & 1.1900 & 4.2337 \\
\hline 2 & 9.8114 & 1.1622 & 9.0964 & 2.3143 & 12.1834 \\
\hline 3 & 9.8114 & 1.1645 & 9.0994 & 2.3143 & 15.8560 \\
\hline 4 & 9.8114 & 1.1619 & 9.1356 & 2.3143 & 6.6741 \\
\hline 5 & 9.8014 & 1.1784 & 9.0483 & 2.0429 & 18.4626 \\
\hline 6 & 9.8014 & 1.1779 & 9.0272 & 2.0429 & 6.7377 \\
\hline 7 & 9.8014 & 1.1729 & 9.0604 & 2.0429 & 19.3306 \\
\hline$\ldots$ & $\ldots$ & $\ldots$ & $\ldots$ & $\ldots$ & $\ldots$ \\
\hline 173 & 9.8971 & 1.0970 & 8.1057 & 0.5257 & 1.5282 \\
\hline
\end{tabular}

Table 1. Codified variables and their uncertainties values (cont.)

\begin{tabular}{|c|c|c|c|c|c|c|}
\hline $\begin{array}{c}\sigma_{R_{W}} \\
\boldsymbol{x}_{\mathbf{1 0}^{-07}}\end{array}$ & $\boldsymbol{P}$ & $\boldsymbol{W}$ & $\sigma_{P}$ & $\sigma_{W}$ & $\theta$ & $\delta U$ \\
\hline 4.11 & 67.478 & 75.853 & 0.124 & 0.734 & 0.4631 & 1.02 \\
\hline 0.79 & 98.048 & 102.216 & 0.112 & 0.675 & 0.4995 & 1.01 \\
\hline 8.67 & 97.547 & 102.216 & 0.112 & 0.675 & 0.4945 & 1.03 \\
\hline 28.51 & 98.048 & 102.216 & 0.112 & 0.675 & 0.4922 & 0.98 \\
\hline 1.36 & 86.021 & 90.278 & 0.116 & 0.700 & 0.4790 & 1.04 \\
\hline 12.43 & 85.018 & 91.273 & 0.117 & 0.698 & 0.4837 & 1.05 \\
\hline 5.87 & 86.021 & 90.278 & 0.116 & 0.700 & 0.4863 & 0.99 \\
\hline$\ldots$ & $\ldots$ & $\ldots$ & $\ldots$ & $\ldots$ & $\ldots$ & $\ldots$ \\
\hline 5.05 & 80.5081 & 22.1319 & 0.118 & 0.883 & 0.7863 & 0.50 \\
\hline
\end{tabular}

Migliavacca and Andrade et al. Multivariate regression ...

Table 2. Model coefficients estimates and model goodness-of-fit parameters

\begin{tabular}{|c|c|c|c|c|c|}
\hline$\beta_{0}$ & $\beta_{1}$ & $\beta_{2}$ & $\beta_{11}$ & $\beta_{22}$ & $\beta_{112}$ \\
\hline$-0,2845$ & 2,8816 & $8,857 E-03$ & $-3,989$ & $-2,75 E-05$ & $3,458 E-03$ \\
\hline$R^{2}$ & 0,9931 & $\chi^{2}$ & 177,1 & $\chi_{\text {red }}^{2}$ & 1,06 \\
\hline
\end{tabular}

Table 3. Covariance and correlation matrices

\begin{tabular}{|c|c|c|c|c|c|}
\hline$\beta_{0}$ & $\beta_{1}$ & $\beta_{2}$ & $\beta_{11}$ & $\beta_{22}$ & $\beta_{112}$ \\
\hline $\mathbf{0 , 0 0 5}$ & $-3,9 \mathrm{E}-04$ & $-2,8 \mathrm{E}-05$ & $4,4 \mathrm{E}-03$ & 0,00 & $3,8 \mathrm{E}-06$ \\
$-0,04$ & $\mathbf{0 , 0 2}$ & $-2,1 \mathrm{E}-05$ & $-2,7 \mathrm{E}-03$ & 0,00 & $2,3 \mathrm{E}-05$ \\
$-0,81$ & $-0,329$ & $\mathbf{2 , 3 E}-\mathbf{0 7}$ & $2,6 \mathrm{E}-05$ & 0,00 & 0,00 \\
0,45 & $-0,178$ & $-0,39$ & $\mathbf{0 , 0 2}$ & 0,00 & 0,00 \\
0,58 & 0,17 & $-0,89$ & 0,42 & $\mathbf{1 , 7 E}-\mathbf{1 2}$ & 0,00 \\
$-0,11$ & $-0,36$ & 0,32 & $-0,737$ & $-0,41$ & $\mathbf{2 , 3 E - 0 7}$ \\
\hline
\end{tabular}

In Figs. 3 - 4 are presented the residuals graphs against the controlled variables, which permit to evaluate the regression model residuals heteroscedasticity degree (Vasconcellos e Portella, 2001).

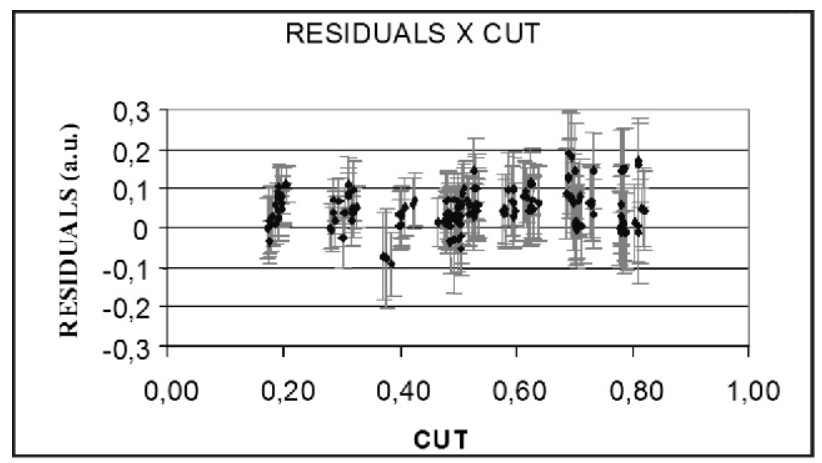

Figure 3. Residuals against .

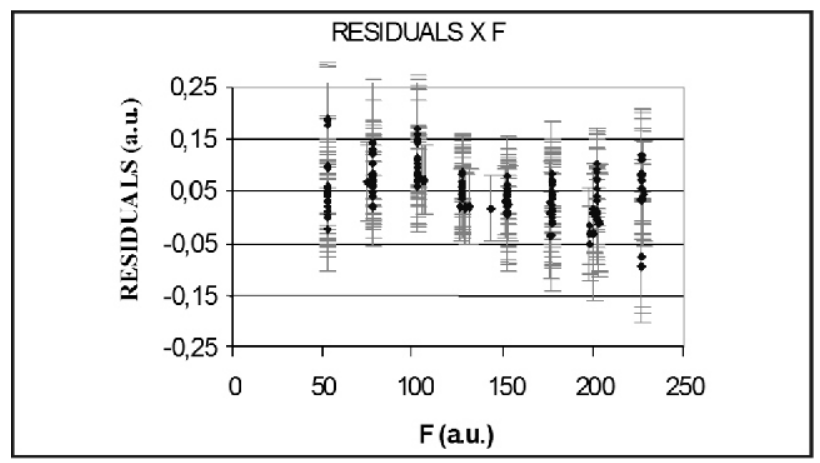

Figure 4. Residuals against $F$.

Through Figs. 5 - 6 it is possible to verify how satisfactorily the theoretic curve fits the experimental data and finally in Fig. 7 is presented the response surface of the separative power $\delta U$ against $F$ and $\theta$ that allows to visualize the $\delta U$ behavior in the ultracentrifugation process and to find the optimum values of the operational controlled variables. 


\section{Tecnologia/Technology}

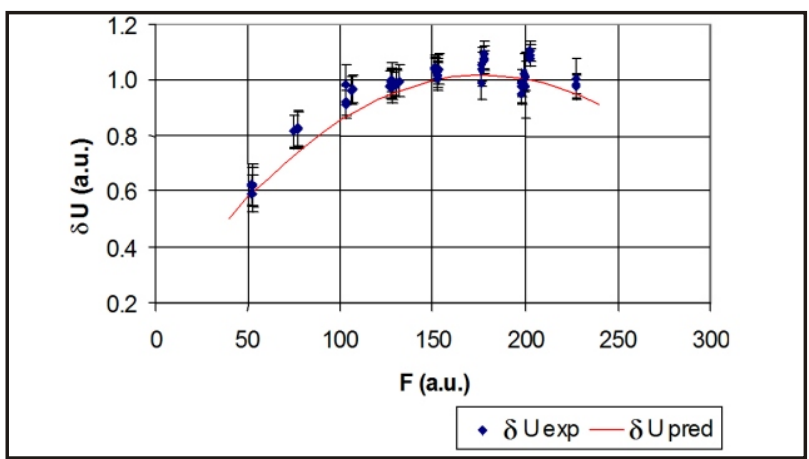

Figure 5. $\delta U$ against $F$.

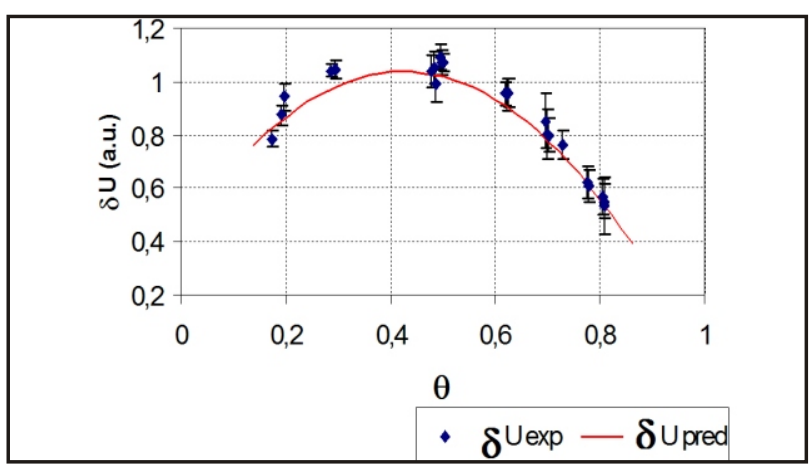

Figure 6. $\delta U$ against.

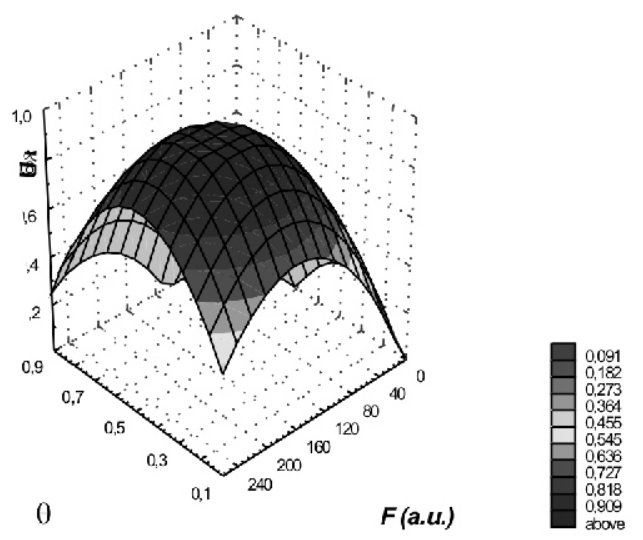

Figure 7. $\delta U$ against $F$ and $\theta$.

\section{CONCLUSIONS}

The least-squares method with covariance matrix was successfully applied in the determination of the ultracentrifuge separative power $\delta U$ fitting curve against experimentally controlled variables. The normalized chisquare was obtained and showed a very reasonable agreement between the $\delta U$ experimental data dispersion and the uncertainties estimated through their covariance matrix. The fitted model was able to explain the experimental data due to the determination coefficient $\left(R^{2}=\right.$ 0,9931). In Figs. 3 - 4, it is possible to verify that there is no visible patterns between the residuals and the control variables and finally through the response curve graphs, Figs. $5-6$, the theoretical model is showed to be reasonably fitted to the experimental data.
Migliavacca and Andrade et al. Multivariate regression ... ACKNOWLEDGEMENTS

The authors acknowledge with gratitude the support of the CTMSP, Centro Tecnológico da Marinha em São Paulo, and IPEN, Instituto de Pesquisas Energéticas e Nucleares, IPEN.

\section{REFERENCES}

Cowan, G. "Statistical data analysis", New York,N.Y.: Oxford University Press, 1998.

Jordan, I. "Separação dos isótopos de urânio pelo processo da centrifugação em fase gasosa", Informação IPEN 3 - IPEN-Inf-3, 1980.

Migliavacca, E. "Modelagem do Comportamento Separativo de Ultracentrífugas por Regressão Multivariada com Matriz de Covariância”, São Paulo, SP, 2004. Dissertação (Mestrado) - Instituto de Pesquisas Energéticas e Nucleares.

Smith, D.L. "Covariance Matrices and Applications to the Field of Nuclear Data. Data and Measurements Series", Argonne National Laboratory. Nov. 1981. ( ANL/NDM-62).

Smith, D.L. "A least-squares computational tool kit. Nuclear Data and Measurements Series", Argonne National Laboratory. Apr. 1993. (ANL/NDM-128).

Vasconcellos, M.T.L.; Portela, M.C. "Índice de Massa Corporal e sua relação com variáveis nutricionais e sócio-econômicas: um exemplo de uso de regressão linear para um grupo de adultos brasileiros. Cad. Saúde Pública”, Rio de Janeiro, nov.-dez. 2001, vol. 17, n.6, p.1425-1436. 